H. Birg

E.-J. Flöthmann

\title{
Langfristige Trends der demographischen Alterung in Deutschland
}

\section{Long-term trends of the demographic aging in Germany}

Zusammenfassung Deutschland verzeichnet zu Beginn des 21 . Jahrhunderts nach Japan die weltweit stärkste demographische Alterung. Sie war zunächst vor allem mortalitätsinduziert, d.h. der deutliche Anstieg der ferneren Lebenserwartung, speziell im höheren Alter, führte zu einer Zunahme der Zahl älterer Menschen. Diese Entwicklung wurde zum Ende des 20. Jahrhunderts durch eine ferti-

Eingegangen: 16. Juli 2002

Akzeptiert: 25. Juli 2002

Prof. Dr. Herwig Birg (

Institut für Bevölkerungsforschung und Sozialpolitik

der Universität Bielefeld

Postfach 100131

33501 Bielefeld, Germany

Tel.: $0521 / 106-5161$ oder -5162

Fax: 05 21/106-60 09

E-Mail: herwig.birg@uni-bielefeld.de

E-Mail: juergen.floethmann@

uni-bielefeld.de litätsinduzierte Alterung noch verstärkt. Der Rückgang der Geburtenrate unter das Bestandserhaltungsniveau seit Ende der 60er Jahre führte zu erheblichen Geburtendefiziten und zu einer nachhaltigen Zunahme des Altenquotienten. Eigene Simulationsrechnungen belegen, dass sich aufgrund der Eigendynamik demographischer Prozesse diese Entwicklung bis zur Mitte des 21. Jahrhunderts weiter verstärken wird. Einerseits wird die Zahl besonders der hochbetagten Bevölkerung um den Faktor 3-3,5 zunehmen, andererseits wird sich der Altenquotient mehr als verdoppeln. Migrationsprozesse haben auf diese Entwicklung nur einen unwesentlichen Einfluss. Ein altersstrukturbedingter Rückgang der Zahl älterer Menschen ab Mitte des 21 . Jahrhundert, wenn die geburtenstarken Nachkriegsjahrgänge verstorben sind, kann die demographische Alterung ebenfalls nicht aufhalten. Allein ein starker und dauerhafter Wiederanstieg der Geburtenraten könnte nach frühestens zwei Generationen (ca. 60 Jahren) zu einer nachhaltigen Reduzierung der demographischen Alterung führen.

Schlüsselwörter Altenquotient Demographische AlterungHochbetagte Bevölkerung Lebenserwartung - Mortalität
Summary At the beginning of the 21st century, Germany is experiencing the highest demographic aging after Japan. The aging process was first initiated by the increase of life expectancy, especially at the age above 60 years which caused an increase in the number of old people. During the next phase (1970-2000) the aging process was additionally affected by decreasing birth rates. Since the end of the 1960s the total fertility rate in Germany has been below the replacement level. As a consequence the number of children is decreasing and the number of old people has been increasing for 30 years. Various population projections published by the Institute for Population Research and Social Policy (IBS) demonstrate that the aging process will intensify during the next 50 years: the number of the oldest old population will triple, and the dependancy ratio will at least double. This process cannot be stopped or considerably diminished by an increasing number of young immigrants. After 2050 the cohorts born after the second world war (the baby boom cohorts) will be dead. But the decreasing number of old people beyond 2050 will not automatically lead to a lower dependancy ratio, because the age group $0-20$ is diminishing continuously. Only a rise of the total fertility rate to the replacement 
level of 2.1 children per women could stop the aging process effectively in the long run (i.e., after two generations or after 60 years, respectively). In this case, the de- pendancy ratio will not double or triple but increase by a factor of 1.5 .

Key words

Demographic aging - life expectancy -

oldest old population -

dependency ratio

(potential support ratio) mortality

\section{Einführung}

Die Wissenschaft unterscheidet drei Arten von Altersbegriffen, erstens das biologisch bedingte Alter der Körperzellen, zweitens das abnutzungs- und krankheitsbedingte Alter der Organe und des menschlichen Körpers als Ganzes und drittens das rechnerisch ermittelte, statistisch-demographische Durchschnittsalter bzw. die Lebenserwartung der Bevölkerung. Die erste Definition ist für die noch offene Frage nach der theoretisch möglichen, maximalen Lebensspanne des Menschen wichtig, auf die zweite und dritte gibt es Antworten in Form von Berechnungen der durchschnittlichen Lebenserwartung und der Trends ihrer Veränderungen. Da die statistisch-demographische Lebenserwartung einer Population als Ganzes als Durchschnitt der Lebenszeiten ihrer individuellen Mitglieder ermittelt wird und die faktische Lebensdauer der einzelnen Menschen identisch ist mit der ihrer Körperzellen, sind alle Erkenntnisse über die maximale Lebensdauer der menschlichen Körperzellen und die diesbezüglichen Fortschritte der Biomedizin auch für die Interpretation und Prognose der demographischen Lebenserwartung einer Population von entscheidender Bedeutung.

Nach den von Leonard Hayflick in Labor-Experimenten mit menschlichen Körperzellen gewonnenen Erkenntnissen soll eine zwischen den Individuen variierende, durch die Biologie vorgegebene Obergrenze der Teilungshäufigkeit der menschlichen Körperzellen existieren (20). Die Zelle stirbt, wenn ihre Erneuerungsfähigkeit durch Teilung erschöpft ist. Auf Grund dieser biologischen Experimente wird die maximale Lebensspanne des Menschen meist auf durchschnittlich 120 Jahre geschätzt (sogenannte "Hayflick-Grenze“). Diese These ist umstritten. Von einigen Demographen wird die Existenz einer nicht übersteigbaren maximalen Obergrenze der menschlichen Lebensspanne trotz der experimentellen Befunde in Zweifel gezogen (30). Diese Demographen berufen sich auf einen anderen Typ von Experimenten mit bestimmten Tieren, bei denen die durchschnittliche Lebensspanne beispielsweise von Fruchtfliegen und Fadenwürmern beträchtlich gesteigert werden konnte, indem jeweils diejenigen Individuen einer Tierpopulation mit überdurchschnittlicher Lebensdauer ausgewählt und dann über mehrere Generationen hinweg gezielt vermehrt wurden.
Die beiden unterschiedlichen Positionen hinsichtlich der Existenz oder Nichtexistenz einer unübersteigbaren Grenze der menschlichen Lebensspanne werden zwischen den Wissenschaftlergruppen außerordentlich kontrovers diskutiert. Eine Entscheidung in der Sache durch naturwissenschaftliches Experimentieren mit Menschen nach Art der Tierversuche scheidet aus. Mit Experimenten, die heute durchgeführt werden, lassen sich aber ohnehin künftige Fortschritte der wissenschaftlichen Erkenntnis nicht antizipieren, so dass eine Klärung der Frage durch Experimente selbst dann nicht möglich wäre, wenn Experimente mit Menschen nicht aus ethischen Gründen abgelehnt werden müssten. Deshalb bleibt die Frage, ob eine Grenze für die menschliche Lebenserwartung existiert, wo sie gegebenenfalls liegt und ob bzw. wie sie hinausgeschoben werden kann, aus prinzipiellen Gründen bis auf weiteres offen.

Im Gegensatz zu der umstrittenen Frage nach der maximalen Lebensspanne sind demographische Erkenntnisse und Aussagen über die durchschnittliche Lebenserwartung bei der Geburt bzw. über die fernere Lebenserwartung in einem bestimmten Alter nicht spekulativ und aus prinzipiellen Gründen unentscheidbar, sondern konkret und nachprüfbar. $\mathrm{Da}$ sich aber in den Trends der Lebenserwartungserhöhung sämtliche Erfolge widerspiegeln, die die persönlichen und die gesellschaftlichen Bemühungen für eine Verbesserung der Gesundheits- und Lebensbedingungen zeitigen, könnten sich die empirisch gemessenen demographischen Lebenserwartungserhöhungen mit ihren langfristigen Wachstumstrends schließlich auch für die theoretische Frage nach den möglichen Grenzen der Lebensspanne des Menschen als immer wichtiger erweisen.

\section{Ursachen, Ausmaß und Arten der demographischen Alterung im 20. Jahrhundert}

Zur Messung der „Alterung“ einer Bevölkerung stehen verschiedene statistische Indikatoren zur Verfügung. Hierzu gehören vor allem

1. die Lebenserwartung im Zeitpunkt der Geburt bzw. die fernere Lebenserwartung in einem bestimmten Alter, 
2. das Medianalter, das so ermittelt wird, dass es die Bevölkerung in zwei Hälften teilt, von denen die eine das Alter über- und die andere es unterschreitet,

3. das erwartete Medianalter einer vorgestellten, fiktiven Gruppe von z. B. 100000 Neugeborenen, das mit den geltenden, in der Sterbetafel festgehaltenen Sterbewahrscheinlichkeiten ermittelt wird (= Medianalter der Sterbetafelbevölkerung),

4. der Altenquotient der Bevölkerung (= Zahl der 60-Jährigen und Älteren auf 100 Menschen im Alter von 20 bis unter 60), für dessen Berechnung auch alternativ die Altersschwellen 15/65 u.a. Intervallgrenzen herangezogen werden,

5. die Prozentanteile der Altersgruppen an der Gesamtbevölkerung, z.B. der Anteil der unter 20-Jährigen, der 20 - bis unter 60 -Jährigen und der über 60-Jährigen sowie

6. die Zahl sowie der Anteil der Betagten und Hochbetagten (meist als 80-Jährige und Ältere definiert) bzw. die Zahl der Hundertjährigen und älteren („Centenarians bzw. „Super-Centenarians“, definiert als Zahl der über 105-Jährigen).

Im 20. Jahrhundert hat die Lebenserwartung in den Industrieländern stark zugenommen, sie stieg z.B. in Deutschland von der Sterbetafel für den Zeitraum $1891 / 1900$ bis zur Sterbetafel $1997 / 99$ bei den Männern von 40,6 auf 74,4 Jahre und bei den Frauen von 44,0 auf 80,6 (35). Es ist daher verständlich, dass sich die meisten Untersuchungen in den letzten Jahrzehnten auf die Lebenserwartung als Maß für die demographische Alterung stützten, obwohl die Lebenserwartung meist nur für das fiktive Kollektiv der Sterbetafelbevölkerung berechnet wird und wesentlich größere Interpretationsprobleme aufwirft, als dies zunächst scheint.

Weniger stark beachtet wurden bisher die absehbaren Veränderungen des Medianalters der Bevölkerung und des Altenquotienten. Ihre Zunahme wird jedoch im 21. Jahrhundert noch größere Ausmaße annehmen als die Lebenserwartungszunahme im 20. Jahrhundert. Das Wachstum des Altenquotienten übertrifft das Wachstum der Lebenserwartung, weil der Anstieg des Altenquotienten von mehreren Faktoren beeinflusst wird, zum einen vom Wachstum der Lebenserwartung, zum anderen von der niedrigen Geburtenrate bzw. von der abnehmenden Größe der nachrückenden Generationen. Sämtliche demographischen Prozesse - insbesondere die Grundprozesse der Fertilität, Mortalität und Migration - die eine Änderung des Altersaufbaus der Bevölkerung bewirken, sind potentielle Ursachen der demographischen Alterung bzw. Verjüngung. Dabei lassen sich vier Typen von Altersstrukturveränderungen unterscheiden.

\section{Demographische Alterung auf Grund niedriger Fertilität}

Durch den Fertilitätsprozess wurde in den letzten Jahrzehnten in den meisten Industrieländern ein starker Rückgang der Geburtenrate (Lebendgeborene pro Frau) und der absoluten Geburtenzahl ausgelöst, der eine Einschnürung am Sockel der Bevölkerungspyramide bewirkte, so dass sich das Medianalter der Bevölkerung z. B. in Deutschland von 1950-2000 von 25 auf 40 Jahre erhöhte. Bis 2050 wurde ein weiterer Anstieg auf über 50 Jahre vorausberechnet (9).

\section{Demographische Alterung auf Grund niedriger Mortalität}

Durch den Mortalitätsprozess änderte sich der Altersaufbau vor allem an der Spitze der Bevölkerungspyramide, wobei im 20. Jahrhundert zwei Etappen $\mathrm{zu}$ unterscheiden sind.

1. In der ersten Etappe, die etwas mehr als die erste Hälfte des 20. Jahrhunderts umfasste, beruhte der hohe Zugewinn an Lebenserwartung primär auf dem Rückgang der Säuglings- und Kindersterblichkeit und auf der Abnahme der Sterblichkeit im jüngeren und mittleren Alter als Folge medizinischer Fortschritte bei der Bekämpfung von Infektionskrankheiten.

2. Im Unterschied dazu ist der Zuwachs an Lebenserwartung in der zweiten Etappe in den letzten Jahrzehnten des 20. Jahrhunderts primär auf die Abnahme der Sterbewahrscheinlichkeiten im höheren Alter zurückzuführen. Diese Abnahme beruht in starkem Maße auf den Fortschritten der Medizin bei der Bekämpfung der Herz- und Kreislauferkrankungen als den häufigsten Todesursachen.

\section{Migrationsinduzierte Verjüngung}

Durch die Einwanderungsüberschüsse vieler Industrieländer, insbesondere Deutschlands, ändert sich die Altersstruktur zunächst in Richtung auf eine Verjüngung, weil die überwiegende Zahl der Zugewanderten auf die Altersgruppe der 25- bis 35-Jährigen entfällt, während das durchschnittliche Alter der Fortgezogenen höher liegt. Die im Alter von z.B. 30 Jahren Zugewanderten gehören jedoch nach drei Jahrzehnten zur Gruppe der 60-Jährigen und Älteren. Die Einwanderung nach Deutschland hat deshalb einen doppelten Effekt: Sie bewirkt kurz und mittelfristig eine Verjüngung der Bevölkerung, langfristig schwächt sich der Verjüngungseffekt jedoch wieder $\mathrm{ab}$ und verkehrt sich in sein Gegenteil. Aus 
den komplizierten gegenläufigen Entwicklungen ergibt sich unter dem Strich nur ein sehr schwacher Netto-Verjüngungseffekt. Wollte man den Anstieg des Altenquotienten in Deutschland durch die Einwanderung jüngerer Menschen stoppen, müssten nach den Vorausberechnungen der UN bis 2050 netto 188 Mio. Menschen nach Deutschland einwandern $(3,40)$.

Neben dem Primäreffekt der Wanderungen auf die Zahl und Altersstruktur der Bevölkerung wird ihr Sekundäreffekt auf die Geburtenbilanz unterschieden, der darauf beruht, dass die Altersstruktur der Ausländer jünger und die Kinderzahl pro Frau höher als bei der deutschen Bevölkerung ist. Die Summe aus Primär- und Sekundäreffekt kann jedoch den Anstieg des Altenquotienten nicht verhindern, sondern nur relativ geringfügig abschwächen.

\section{Transformationsbedingte Alterung}

$\mathrm{Zu}$ den fertilitäts-, mortalitäts- und migrationsbedingten Komponenten der demographischen Alterung kommt ein Faktor hinzu, der sich aus dem $\mathrm{Zu}$ sammenwirken einer niedrigen Fertilität und einer hohen Lebenserwartung ergibt. Das daraus entstehende Geburtendefizit bzw. die Bevölkerungsschrumpfung (ohne Kompensation durch Einwanderungen) hat einen Typ der demographischen Alterung hervorgebracht, der die meisten Industrieländer und zunehmend auch die Entwicklungsländer betrifft $(1,2)$. Die Industrieländer vollzogen im Prozess der demographischen Transformation einen Übergang von der vorindustriellen Phase mit hoher Geburten- und Sterberate zur industriellen und postindustriellen Phase mit niedriger Geburten- und Sterberate $(14,22)$. Am Anfang des Transformationsprozesses herrscht die mortalitätsinduzierte Alterung vor, in der mittleren und späten Phase des demographischen Übergangs kam die fertilitätsinduzierte Alterung hinzu. In der Frühphase des demographischen Transformationsprozesses bewirkte der Rückgang der Säuglings- und Kindersterblichkeit in vielen Ländern vorübergehend eine Verjüngung statt eine Alterung der Bevölkerung (24).

\footnotetext{
${ }^{1}$ Der Human Development Index ist das gewogene Mittel aus den Variablen Lebenserwartung, Pro-Kopf-Einkommen und Ausbildungsniveau der Bevölkerung. Siehe (39)

${ }^{2} \mathrm{Da}$ der Human Development Index die Lebenserwartung als Teilindikator enthält, sollte die Lebenserwartung nicht mit dem HDI, sondern mit einem anderen Indikator für den Entwicklungsstand korreliert werden, z.B. mit dem Education-Income-Index (=HDI exclusive Lebenserwartung)
}

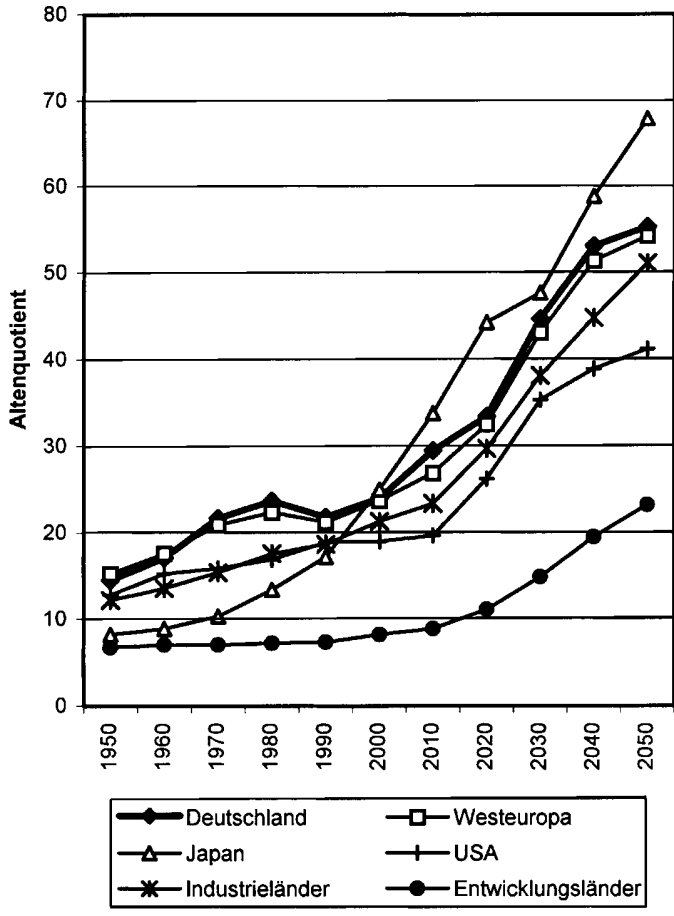

Abb. 1 Entwicklung des Altenquotienten (=Zahl der 65-Jährigen und Älteren auf 100 Menschen im Alter von 15-64) von 1950-1955 bis 1995-2000 und Projektionsrechnungen bis 2050. Quelle: H. Birg, IBS, Universität Bielefeld. Daten: UN (Ed.), World Population Prospects, 1998 Revision, New York 1999

Zwischen dem Entwicklungsniveau eines Landes (gemessen z.B. durch den Human Development Index (HDI) der Vereinten Nationen ${ }^{1}$ und der Zahl der Lebendgeborenen pro Frau besteht ein gegenläufiger Zusammenhang. Im Unterschied dazu ist die Lebenserwartung eines Landes umso größer, je höher der erreichte Entwicklungsstand ist (gemessen z.B. durch den Education-Income-Index). ${ }^{2}$ Aus diesen grundlegenden Zusammenhängen ergibt sich, dass sich die fertilitätsinduzierte und die mortalitätsinduzierte Alterung im 21. Jahrhundert umso stärker fortsetzen werden, je rascher der weltweite Entwicklungsprozess voranschreitet (Abb. 1).

\section{Vorausberechnungen für das 21. Jahrhundert}

\section{Auswirkungen des generativen Verhaltens auf die demographische Alterung}

Die zukünftige Entwicklung der demographischen Alterung ist sowohl von Einflüssen abhängig, die bereits vor Jahrzehnten wirksam waren, als auch von demographisch relevanten Veränderungen in der $\mathrm{Zu}$ kunft. Der Geburtenboom nach dem Zweiten Welt- 
Abb. 2 im Jahr 2000

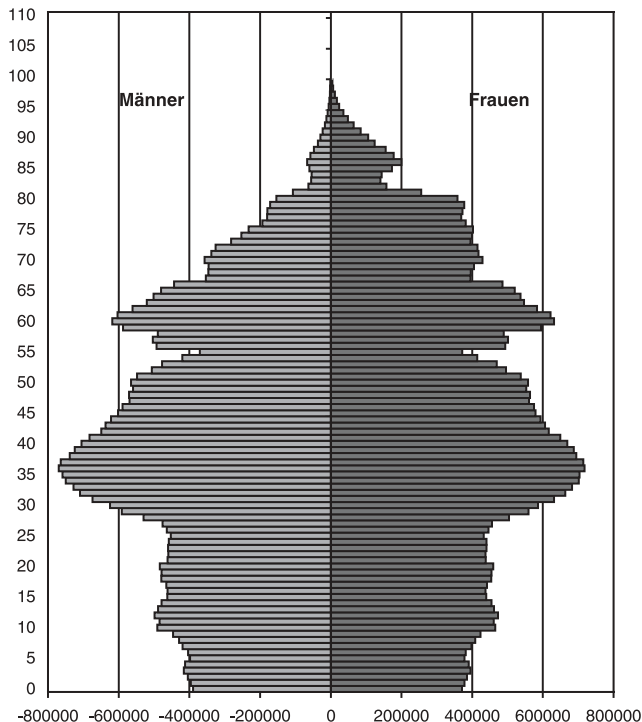

Altersstruktur der Bevölkerung Deutschlands im Jahr 2050

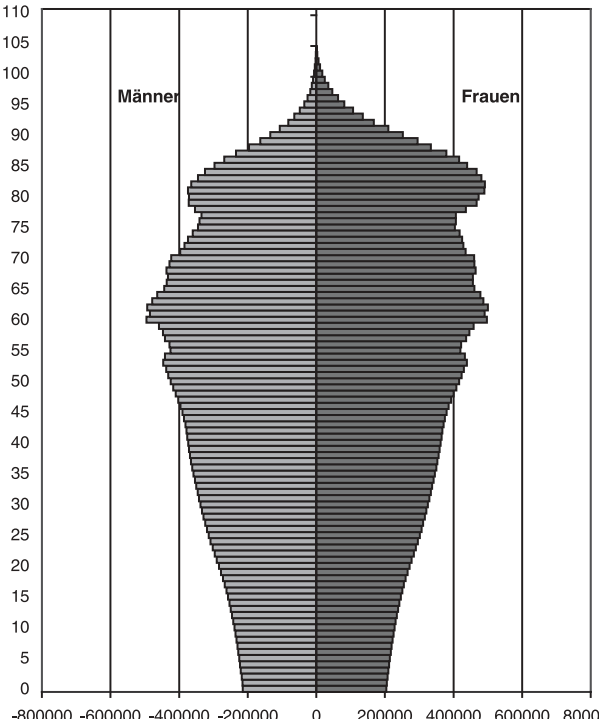

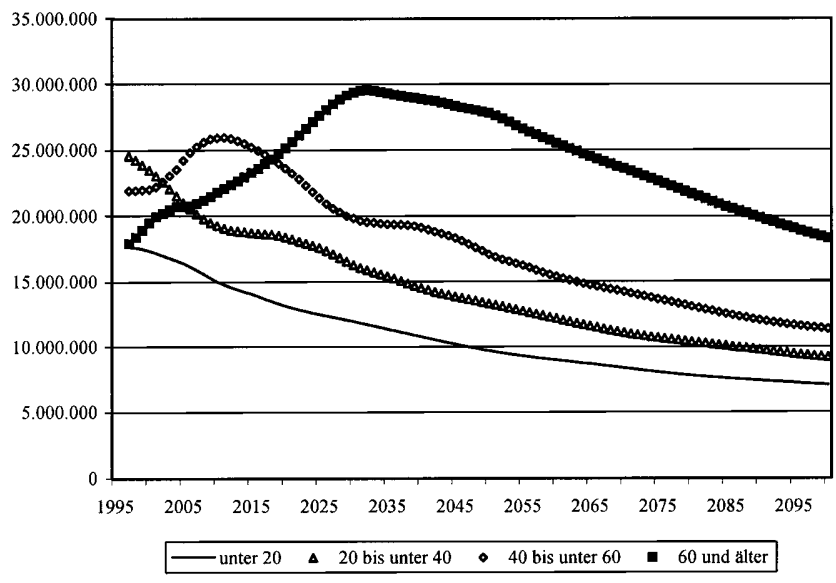

Abb. 3 Vorausgeschätzte Zahl der Gesamtbevölkerung in Deutschland nach Altersklassen

krieg, der starke Geburtenrückgang Ende der 60er Jahre sowie der Rückgang in Folge der Wiedervereinigung sind historische Ereignisse, die die Bevölkerungsstruktur Deutschlands und die Dynamik der demographischen Alterung in den nächsten Jahrzehnten maßgeblich bestimmen werden. Die künftigen Entwicklungen der Lebenserwartung, der durchschnittlichen Kinderzahl und der Außenwanderungen können den durch die genannten Ereignisse bereits weitgehend vorprogrammierten Alterungsprozess nur begrenzt beeinflussen. Dies gilt auch für Änderungen des generativen Verhaltens, die sich erst nach zwei Generationen, d.h. mit einer Verzögerung von Jahrzehnten voll auswirken.
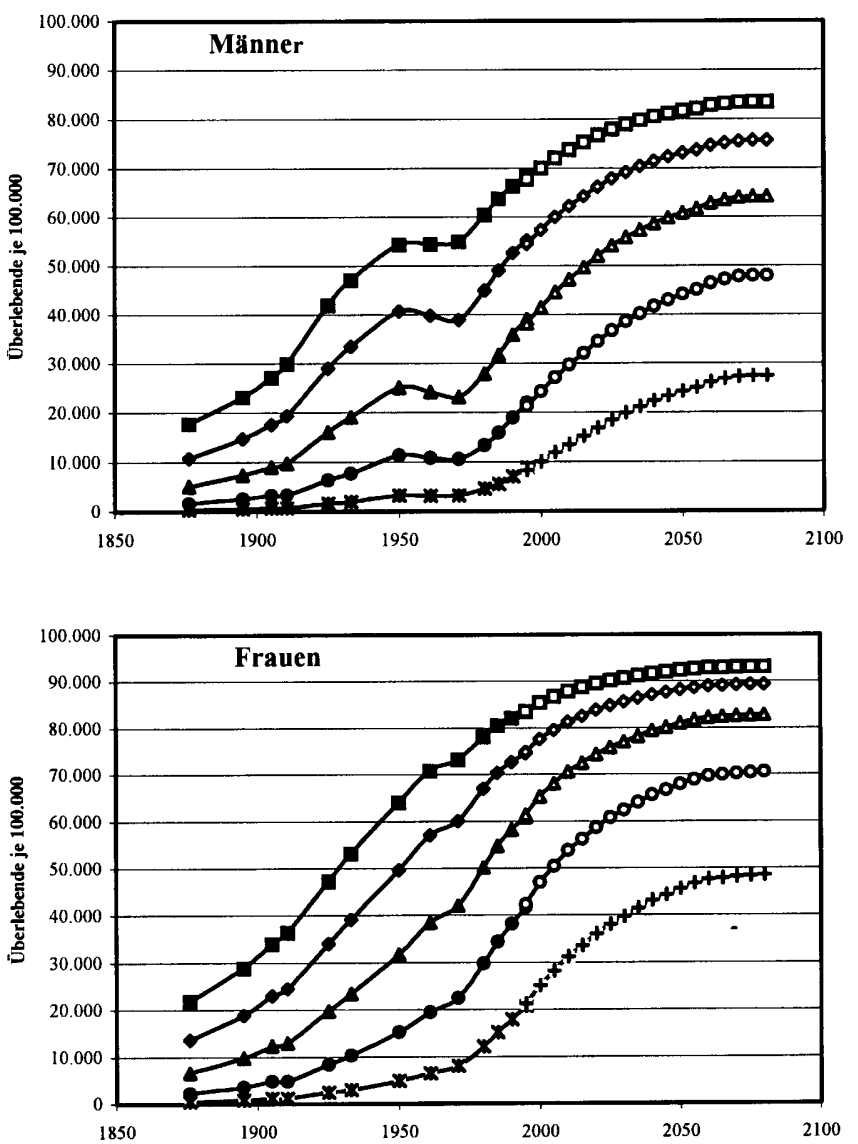

Abb. 4 Anteil der bis zum Alter 70, 75, 80, 85 und 90 Jahre Überlebenden nach den Sterbetafeln von $1871 / 81$ bis $1994 / 96$ mit Projektionen bis 2080. Quelle: Birg/Flöthmann, Demographische Projektionsrechnungen für Die Rentenreform 2000, Bielefeld 2000 


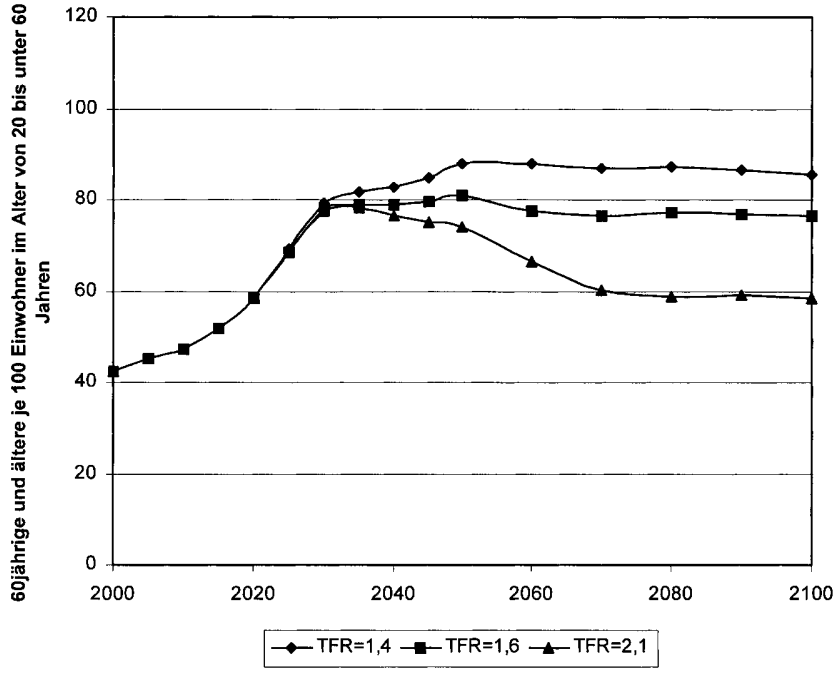

Abb. 5 Entwicklung des Altenquotienten in Abhängigkeit von der künftigen Entwicklung der durchschnittlichen Kinderzahl

Aufgrund der bisherigen Entwicklung, d.h. vor allem wegen der seit drei Jahrzehnten deutlich unter dem Bestandserhaltungsniveau liegenden durchschnittlichen Kinderzahl, ist die Entwicklung der Alterung für die nächsten 30 Jahre unabwendbar vorgegeben (Abb. 2-4). Der Geburtenrückgang Ende der 60 er Jahre und die dann auf niedrigem Niveau verbleibende Geburtenzahl von rd. 1,4 Lebendgeborenen pro Frau führten zunächst zu einem Rückgang der Zahl jüngerer Menschen. In den nächsten Jahrzehnten wird sich dieser Rückgang bei der 20- bis unter 60-jährigen Bevölkerung fortsetzen. Eine Kompensation dieses Rückgangs durch Zuwanderungen vor allem junger Migranten war trotz teilweise hoher Wanderungsgewinne bereits in der Vergangenheit nicht möglich.

Selbst ein sofortiger Wiederanstieg der Kinderzahl auf einen Wert von 2,1 Kindern je Frau auf das sogenannte Bestandserhaltungsniveau würde die sich abzeichnende Verdoppelung des Altenquotienten nicht aufhalten können. Ob die Kinderzahl während der nächsten 30 Jahre auf dem akutellen Wert konstant bleibt oder auf 2,1 Kinder ansteigt, ist für die Entwicklung bis 2030 zunächst unerheblich (Abb. 5). Auch für die folgenden 20 Jahre (bis 2050) ist die Höhe der Kinderzahl für die demographische Alterung relativ unbedeutend. Der Altenquotient wird bis 2030 auf ca. 80 , bis 2050 auf ungefähr 78 bis 84 zunehmen. Bei einer konstanten Kinderzahl von 1,4 oder bei einem leichten Wiederanstieg auf 1,6 Kinder je Frau würde ungefähr ab 2050 eine Konsolidierung der demographischen Alterung auf dem erreichten hohen Niveau eintreten. Dies bedeutet, dass unter diesen Annahmen bis zum Ende des 21. Jahr- hunderts nicht mit einer Reduzierung des Altenquotienten gerechnet werden kann.

Nur ein sofortiger, rein theoretischer Wiederanstieg der durchschnittlichen Kinderzahl auf 2,1 Kinder je Frau könnte, allerdings erst nach 2050, $\mathrm{zu}$ einem deutlichen und vor allem nachhaltigen Rückgang des Altenquotienten beitragen. Erst ab 2060, d.h. nach ungefähr zwei Generationen, würden die Auswirkungen eines Wiederanstiegs der Kinderzahl auf die demographische Alterung deutlich. Der Altenquotient würde in diesem Fall ab 2070 ungefähr 60 betragen. Kein anderer Effekt, von einem extrem starken Rückgang der Lebenserwartung einmal abgesehen, könnte die demographische Alterung in diesem Umfang und derart nachhaltig reduzieren, wobei allerdings das reduzierte Niveau des Altenquotienten immer noch um rd. die Hälfte über dem heutigen Niveau (rd. 40\%) läge.

\section{Lebenserwartung und demographische Alterung}

Die zukünftige Entwicklung der Zahl älterer Menschen und des Altenquotienten werden einerseits von der aktuellen Bevölkerungsstruktur, hier insbesondere von den geburtenstarken Jahrgängen, und andererseits von der weiteren Entwicklung der Lebenserwartung bestimmt. Der Anstieg der Lebenserwartung während der letzten Jahrzehnte beruhte in erster Linie auf einem Anstieg der ferneren Lebenserwartung im höheren Alter. Dieser Sachverhalt erfordert im Rahmen von Bevölkerungsvorausschätzungen eine besonders differenzierte Modellierung des zukünftigen Verlaufs der alters- und geschlechts-

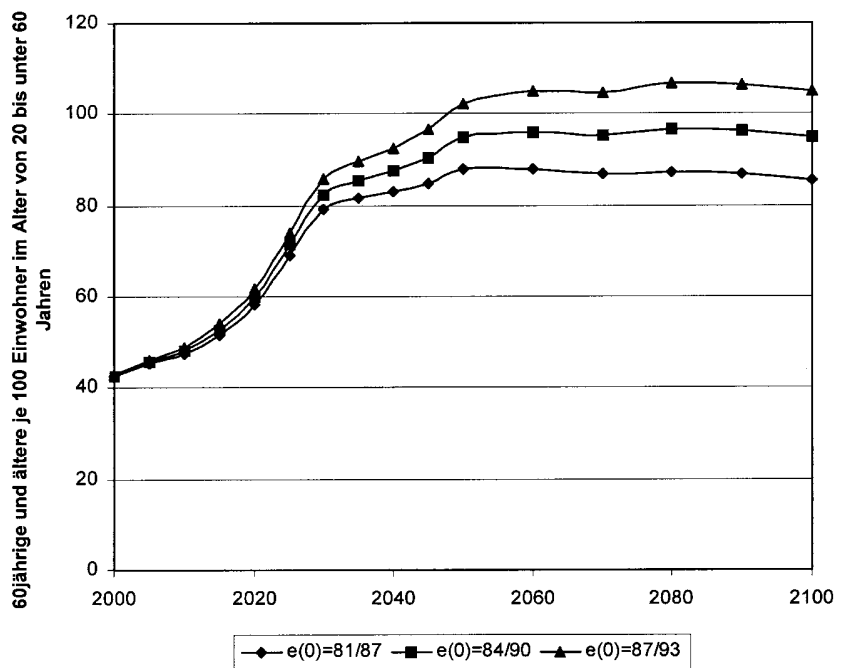

Abb. 6 Entwicklung des Altenquotienten in Abhängigkeit von der künftigen Entwicklung der Lebenserwartung 
spezifischen Sterbe- bzw. Überlebenswahrscheinlichkeiten. Solange wie ein weiterer Anstieg der ferneren Lebenserwartung im höheren Alter zu verzeichnen ist, wird sich die Zahl der älteren Menschen besonders stark erhöhen. Aber auch im Fall einer konstanten Lebenserwartung wird sich die demographische Alterung allein aufgrund des genannten Altersstruktureffekts weiter intensivieren.

Durch das Zusammenwirken beider Komponenten, des Altersstruktureffekts und einer zunehmenden Lebenserwartung, wird sich die Zunahme der demographischen Alterung in jedem Fall beschleunigen (Abb. 6). Die Verdoppelung des Altenquotienten bis zum Jahr 2030 wird zwar zunächst relativ unabhängig vom weiteren Verlauf der Lebenserwartung erfolgen. Aber in den folgenden beiden Jahrzehnten bis 2050 wird sich der Anstieg der Lebenserwartung deutlich auswirken. Danach konsolidiert sich die Entwicklung des Altenquotienten auf dem dann erreichten hohen Niveau. Bei einem starken Anstieg der Lebenserwartung (auf 87 Jahre bei Männern und auf 93 Jahre bei Frauen) würde der Altenquotient auf einen Wert von ca. 105 wachsen. Unter Konstanthaltung aller anderen Parameter führt eine Erhöhung der Lebenserwartung um 3 Jahre langfristig im Durchschnitt zu einer Erhöhung des Altenquotienten um ca. 10 Prozentpunkte.

Obwohl die geburtenstarken Jahrgänge in der zweiten Hälfte des 21. Jahrhunderts verstorben sein werden, ist in diesem Zeitraum kein Rückgang der Alterung zu erwarten. Dieser Effekt kann, wie im Zusammenhang mit den Auswirkungen der Fertilität dargestellt, erst dann eintreten, wenn die nachwachsenden Generationen wieder deutlich stärker besetzt sein werden. Allein ein Rückgang der Zahl älterer Menschen kann die Alterung also noch nicht aufhalten, es muss ein deutlicher Anstieg der Zahl der Jüngeren hinzukommen.

\section{Konsequenzen der Einwanderungen für die demographische Alterung}

Seit Anfang der 70er Jahre des 20. Jahrhunderts verzeichnete Deutschland ausschließlich Geburtendefizite, so dass die Bevölkerungszahl ohne diese Wanderungsgewinne bereits seit längerem zurückgegangen wäre. Während der 80er und 90er Jahre konnten die Geburtendefizite in Deutschland durch erhebliche Wanderungsgewinne teilweise ausgeglichen oder sogar überkompensiert werden. Durch den migrationsbedingten Zuwachs an meist jungen Menschen wurde aber nicht nur die Bevölkerungszahl relativ konstant gehalten, sondern auch die demographische Alterung konnte, zumindest vorübergehend, abgeschwächt werden. Dieser günstige Einfluss der Wan-

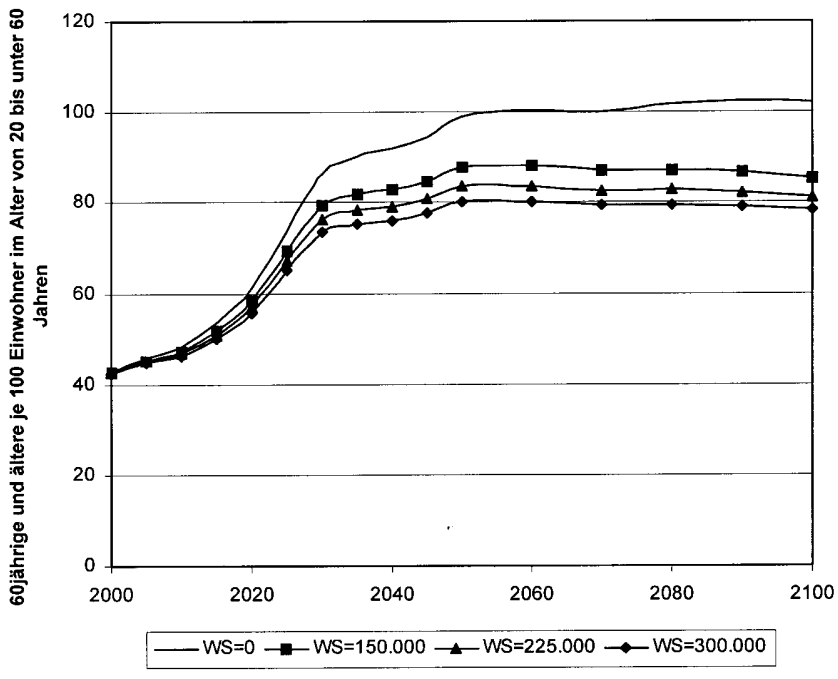

Abb. 7 Entwicklung des Altenquotienten in Abhängigkeit von der künftigen Entwicklung der Außenmigration

derungen auf die Bevölkerungsentwicklung Deutschlands war nur möglich, weil einerseits die Geburtendefizite und die Alterung noch relativ gering und andererseits die Wanderungsgewinne vergleichsweise hoch waren.

Diese demographisch vorteilhafte Konstellation ist zukünftig auch bei stark zunehmenden Wanderungsgewinnen nicht $\mathrm{zu}$ erwarten, da die Eigendynamik der demographischen Entwicklung zu einer exponentiellen Zunahme der Geburtendefizite und damit $\mathrm{zu}$ einer außerordentlich starken Verschiebung der Altersstruktur hin $\mathrm{zu}$ einer zuvor nicht dagewesen demographische Alterung führen wird. Die bereits erwähnten Simulationsrechnungen der Vereinten Nationen für zahlreiche Industriestaaten zeigen, dass zur Konstanz des Altenquotienten auf dem aktuellen Niveau in Deutschland bis zum Jahr 2050 eine Nettozuwanderung in Höhe von 188 Mio. Menschen erforderlich wäre (40). Das würde einem durchschnittlichen Wanderungsgewinn in Höhe von 3,76 Mio. Menschen jährlich entsprechen.

Der nur sehr begrenzte Einfluss der Migration auf die Entwicklung der demographischen Alterung wird aus den in Abbildung 7 dargestellten Szenarien deutlich. Ein jährlicher Wanderungsgewinn von durchschnittlich 300000 Menschen (das wäre bis 2100 eine Nettozuwanderung von 30 Mio. Menschen), würde im Vergleich $\mathrm{zu}$ der Variante mit einem jährlich ausgeglichenem Wanderungssaldo lediglich $\mathrm{zu}$ einem um ungefähr 20 Prozentpunkte niedrigeren Altenquotienten führen. Im ersten Fall würde der Altenquotient langfristig bei ungefähr 80 liegen, im zweiten Fall bei ungefähr 100. Dies bedeutet, dass selbst bei außerordentlich starken konstan- 
ten Wanderungsgewinnen eine nachhaltige Reduzierung der demographischen Alterung nicht erreicht werden kann.

Vergleicht man die Auswirkungen von Mortalität, Fertilität und Migration auf die demographische Alterung anhand der skizzierten Modellrechnungen, wird deutlich, dass die einzige langfristige Chance $\mathrm{zu}$ einer nachhaltigen Reduzierung des Altenquotienten in einem dauerhaften Wiederanstieg der durchschnittlichen Kinderzahl pro Frau besteht.

\section{Bedeutung unterschiedlicher Altersabgrenzungen bei Altenquotienten}

Zur Beurteilung der demographischen Alterung finden unterschiedlich abgegrenzte Altenquotienten Anwendung. In Abbildung 8 sind Altenquotienten mit einer oberen Intervallgrenze von $60,65,70$ und 75 Jahren dargestellt. Die verschiedenen Abgrenzungsvarianten sind vor allem von Bedeutung im Zusammenhang mit Aussagen und Analysen der Konsequenzen einer längeren Lebensarbeitszeit. Die hier bislang berücksichtigte Abgrenzung, nach der die Zahl der 60-jährigen und älteren Bevölkerung auf 100 Personen im Alter von 20 bis unter 60 bezogen wurde, führt in einer mittleren Variante $\mathrm{zu}$ einem

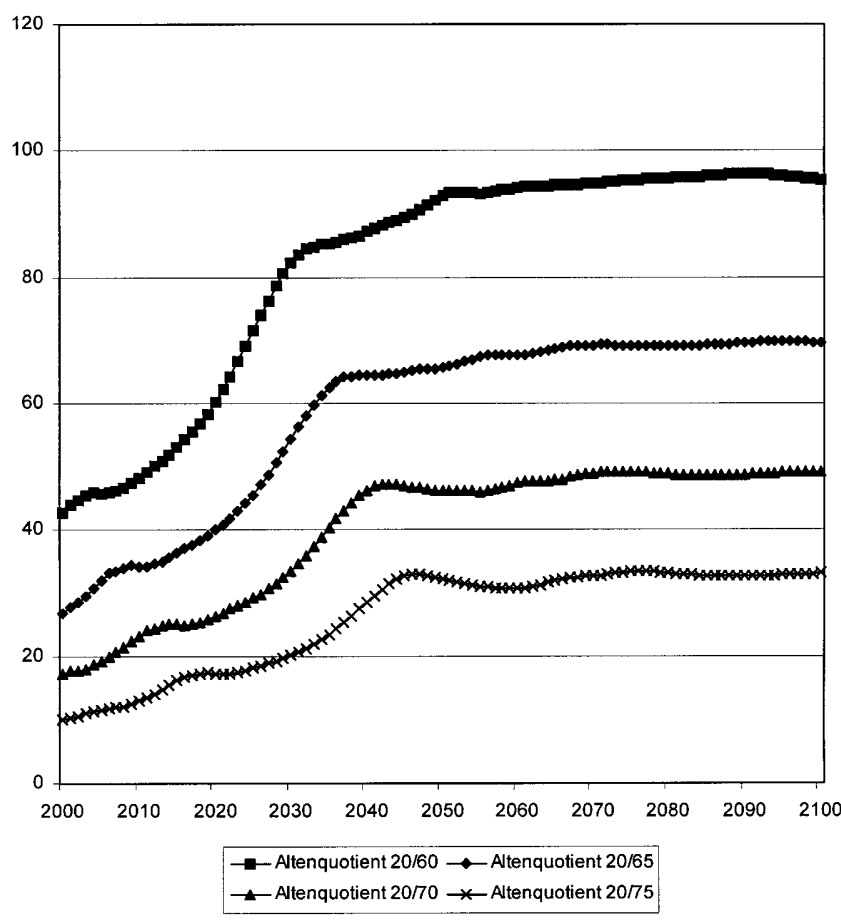

Abb. 8 Entwicklung des Altenquotienten bei unterschiedlicher Abgrenzung der Altersklassen

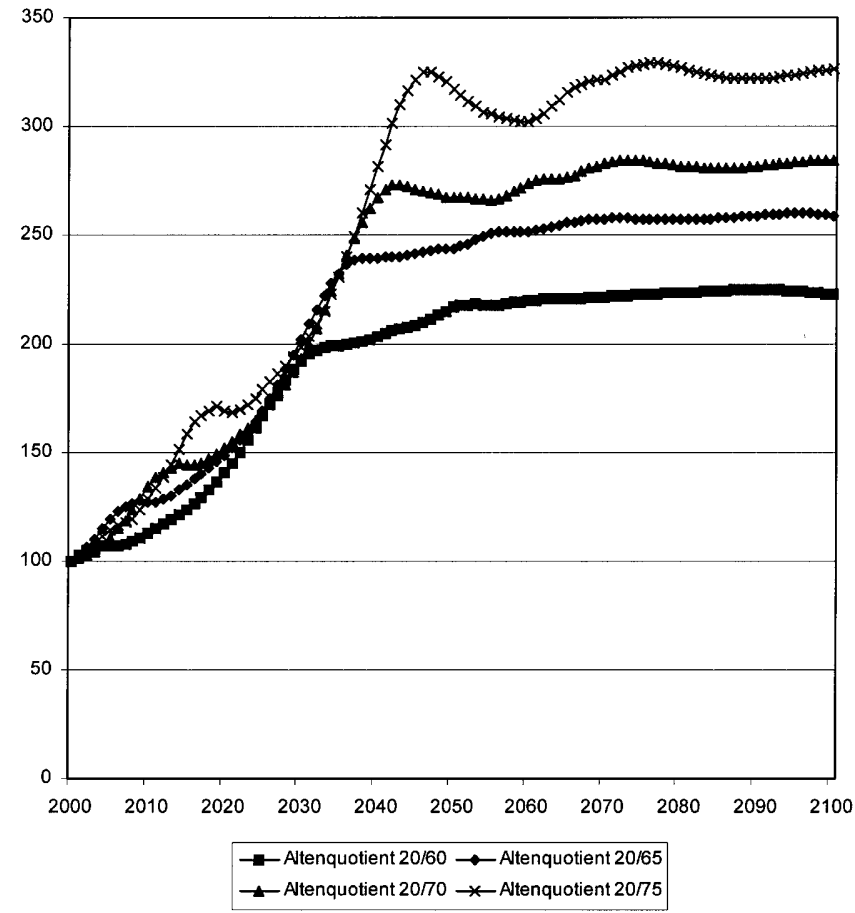

Abb. 9 Entwicklung des Altenquotienten bei unterschiedlicher Abgrenzung der Altersklassen $(2000=100)$

Anstieg von 39 im Jahr 1998 bzw. von 42 im Jahr 2001 auf 90-95 im Jahr 2050. Würde als Altersgrenze nicht 60 , sondern 65 gewählt, dann würde der Altenquotient aktuell bei 27 und langfristig bei 70 liegen. Eine weitere Erhöhung der Altersgrenze um 5 Jahre auf 70 würde langfristig $\mathrm{zu}$ einem Altenquotienten in Höhe von 50 führen. Nur eine Erhöhung der oberen Altersgrenze von 60 auf ca. 72 Jahre würde das Niveau des Altenquotienten langfristig auf dem aktuellen Niveau von 42 stabilisieren.

In diesem Zusammenhang ist aber zu berücksichtigen, dass eine Erhöhung der oberen Intervallgrenze zwar zu einem absolut niedrigeren Altenquotienten führt, der relative Anstieg des Altenquotienten würde aber bei höheren Altersgrenzen umso stärker ausfallen (Abb. 9). Während sich der normalerweise verwendete Altenquotient mit einer oberen Altersgrenze von 60 Jahren langfristig um ca. 120\% erhöht, steigt der Altenquotient mit einer oberen Altersgrenze von 75 Jahren langfristig um über $220 \%$.

\section{Differentielle demographische Alterung}

Die demographische Alterung kennzeichnet eine Population als Ganzes. Differenziert man die Population in Teilpopulationen, erhält man in der Regel für 
jede Teilpopulation unterschiedliche Analyseergebnisse für die demographische Alterung. Wird eine Population z.B. nach Geburtsjahrgängen untergliedert (im Folgenden kurz „Kohorten“), dann tritt die demographische Alterung in einem von Kohorte $\mathrm{zu}$ Kohorte unterschiedlichen Zuwachs der Lebenserwartung in Erscheinung. Zusätzlich zur Differenzierung nach Kohorten ist eine Differenzierung der demographischen Alterung nach sozialen Gruppen sowie nach Regionen aufschlussreich. Die drei Klassifikationskriterien Kohorte, soziale Gruppe und Region lassen sich gedanklich auch kombinieren, allerdings sind die für empirische Analysen benötigten Daten in der Regel nicht verfügbar.

Wird die Lebenserwartung als Maß für die demographische Alterung verwendet, dann beziehen sich die entsprechenden Daten meist auf ein bestimmtes Kalenderjahr, nicht auf bestimmte Kohorten. Die vom Statistischen Bundesamt in den Sterbetafeln veröffentlichte Lebenserwartung für die verschiedenen Kalenderjahre ist eine Art Durchschnitt für die in dem betreffenden Jahr gleichzeitig lebenden $\mathrm{rd}$. 110 Kohorten. So lässt sich z.B. die Aussage, dass die Lebenserwartung der Frauen in Deutschland im Jahr 1999 80,6 Jahre beträgt, als Lebenserwartung interpretieren, die für eine fiktive Durchschnitts- oder Querschnittskohorte gilt, die sich aus den im Jahr 1999 gleichzeitig lebenden 110 Frauenjahrgängen zusammensetzt. Diese Art der Berechnung wird als Querschnittsanalyse bezeichnet, wobei sich der Begriff Querschnitt auf die 110 gleichzeitig lebenden Jahrgänge bezieht. Bei einer im Zeitablauf tendenziell sinkenden Mortalität ist die Lebenserwartung für die jüngeren Kohorten innerhalb der fiktiven Durchschnittskohorte höher als 80,6 Jahre, für die älteren niedriger.

Aussagen über die von Kohorte zu Kohorte variierende demographische Alterung setzen Längsschnittoder Kohortenanalysen der Mortalität und Lebenserwartung und entsprechende Prognosen voraus. Derartige Analysen wurden von verschiedenen Autoren veröffentlicht (z.B. 8-10, 17). Berechnet man die demographische Alterung auf der Basis der Längsschnitt-Analyse der Lebenserwartung, treten zwei wichtige Sachverhalte deutlich hervor:

1. Der prozentuale Zuwachs der ferneren Lebenserwartung in einem bestimmten Alter ist für Menschen, die bereits ein hohes Lebensalter erreicht haben, besonders groß. Da die Säuglings- und Kindersterblichkeit bereits extrem niedrig ist, konzentrieren sich weitere Zuwächse der Lebenserwartung vor allem auf Mortalitätsabnahmen im hohen und höchsten Alter.

2. Bevölkerungsprognosen beruhen stets auf bestimmten Annahmen über die Entwicklung der Lebenserwartung. So wird z.B. in der sogenannten
„9. koordinierten Bevölkerungsvorausberechnung“ des Statistischen Bundesamtes ein Zuwachs von rd. 5 Jahren bis 2050 vorausgesetzt (37). Ein gegebener Zuwachs von z.B. 5 Jahren lässt sich jedoch mit unterschiedlichen - im Prinzip mit unendlich vielen - Annahmen über die Abnahme der Sterbewahrscheinlichkeiten in den einzelnen Altersgruppen modellieren. Dabei hat sich gezeigt, dass die demographische Alterung, gemessen am Anstieg der Zahl der Hochbetagten, in den Bevölkerungsvorausberechnungen des Statistischen Bundesamtes im Vergleich z.B. zu den Ergebnissen der Verfasser niedriger ist. Der Unterschied beruht darauf, dass die Lebenserwartungsprognosen des Statistischen Bundesamtes auf der Querschnittsanalyse aufbauen, mit der der Zuwachs der ferneren Lebenserwartung tendenziell niedriger vorausgeschätzt wird als mit der Längsschnitt-Analyse.

Methodische Unterschiede sind auch bei einer Differenzierung der demographischen Alterung nach Bevölkerungsgruppen und/oder nach Regionen von großer Bedeutung: So wurden beträchtliche Sterblichkeitsunterschiede nach Berufen nachgewiesen (27). Die Sterblichkeit differiert auch nach dem Familienstand, so hat das Statistische Bundesamt z.B. für den Zeitraum 1960/62 spezielle Sterbetafeln und Lebenserwartungsberechnungen für Ledige, Verheiratete, Verwitwete und Geschiedene publiziert (siehe auch 18, 23). Neuere Untersuchungen lassen vermuten, dass die sozio-ökonomischen Unterschiede der Lebenserwartung bisher generell unterschätzt wurden. So haben beispielsweise Untersuchungen für Finnland ergeben, dass sich die Lebenserwartungsunterschiede zwischen den sozioökonomischen Gruppen im Zeitablauf erheblich vergrößern (42).

Die Unterscheidung der demographischen Alterung nach sozioökonomischen Gruppen hat dann eine besonders hohe Aussagekraft, wenn zusätzlich nach Regionen differenziert wird. In den älteren Sterbetafeln des Statistischen Bundesamtes für die 60er und 70er Jahre wurde deshalb die Lebenserwartung nicht nur nach dem Familienstand, sondern zusätzlich nach Gemeindegrößenklassen untergliedert. Nach Stadt- und Landkreisen differenzierte Schätzungen der Lebenserwartung werden seit 1998 auch vom Bundesamt für Bauwesen und Raumordnung veröffentlicht (12). Danach streute die mittlere Lebenserwartung der Männer zwischen den Stadtund Landkreisen im Jahr 1998 im Intervall von 71,6 und 78,0 und die der Frauen zwischen 78,1 und 83,2 .

Aussagen über regionale Sterblichkeitsunterschiede sind jedoch insofern problematisch, als sie sich auf eine Regionsbevölkerung beziehen, deren $\mathrm{Zu}$ sammensetzung in starkem Maße auf Binnen- und 
Außenwanderungen beruht. Im Durchschnitt entfallen auf jede Geburt in einem Stadt- und Landkreis vier Zuzüge, auf jeden Sterbefall rd. drei bis vier Fortzüge. Dies bedeutet, dass die Lebenserwartungsunterschiede nicht einfach die Region beschreiben, für deren Regionsbevölkerung sie gemessen wurden, sondern auch die Herkunftsregionen charakterisieren, aus denen die Regionsbevölkerung in die betreffende Region zugezogen ist. Daraus resultieren nicht nur erhebliche statistische Fehlerrisiken, sondern auch prinzipielle Probleme der Interpretation der gemessenen regionalen Lebenserwartungsunterschiede.

Wesentlich zuverlässiger sind Aussagen über die regionalen Unterschiede der demographischen Alterung, die sich aus folgender Differenzierung ergeben: In Regionen, in denen der Wanderungssaldo älterer Menschen positiv ist, ergibt sich eine „aktive demographische Alterung durch Wanderungen", in Regionen, in denen der Wanderungssaldo jüngerer Menschen negativ ist, eine "passive“ Alterung. Auf dem stufenweisen Prozess der Urbanisierung und Suburbanisierung der 60er und 70er Jahre folgen phasenverschoben regionale Alterungsprozesse, wenn die ursprünglich junge zugezogene Bevölkerung z.B. in den Randzonen der Ballungsgebiete die Altersschwelle um 60 erreicht und überschreitet. Die Grundmuster regionaldemographischer Prozesse werden von speziellen Teilprozessen überlagert, durch die sich die demographische Alterung in bestimmten Siedlungstypen besonders stark intensivieren kann. Hierzu gehören vor allem die Migrationsprozesse älterer Menschen, die sich häufig in Bädern niederlassen oder landschaftlich attraktive Gebiete als Wohnstandorte wählen (6).

\section{Entwicklung der Langlebigkeit und Auswirkungen auf die Zahl der Hochbetagten}

Während in der ersten Hälfte des 20. Jahrhunderts der Anstieg der Lebenserwartung in Deutschland noch $\mathrm{zu}$ einem großen Teil auf einer Reduzierung

Tab. 1 Zunahme der Lebenserwartung bei der Geburt und der ferneren Lebenserwartung von Männern und Frauen in Deutschland zwischen 1949/51 und $1997 / 99$

\begin{tabular}{lll}
\hline $\begin{array}{l}\text { Lebenserwartung } \\
\text { im Alter }\end{array}$ & $\begin{array}{l}\text { Männliche Bevölkerung } \\
(\%)\end{array}$ & $\begin{array}{l}\text { Weibliche Bevölkerung } \\
(\%)\end{array}$ \\
\hline 0 & 15,3 & 17,7 \\
60 & 17,3 & 33,4 \\
70 & 23,4 & 44,8 \\
80 & 31,9 & 50,3 \\
\hline
\end{tabular}

Berechnet nach Daten des Statistischen Bundesamtes (35)

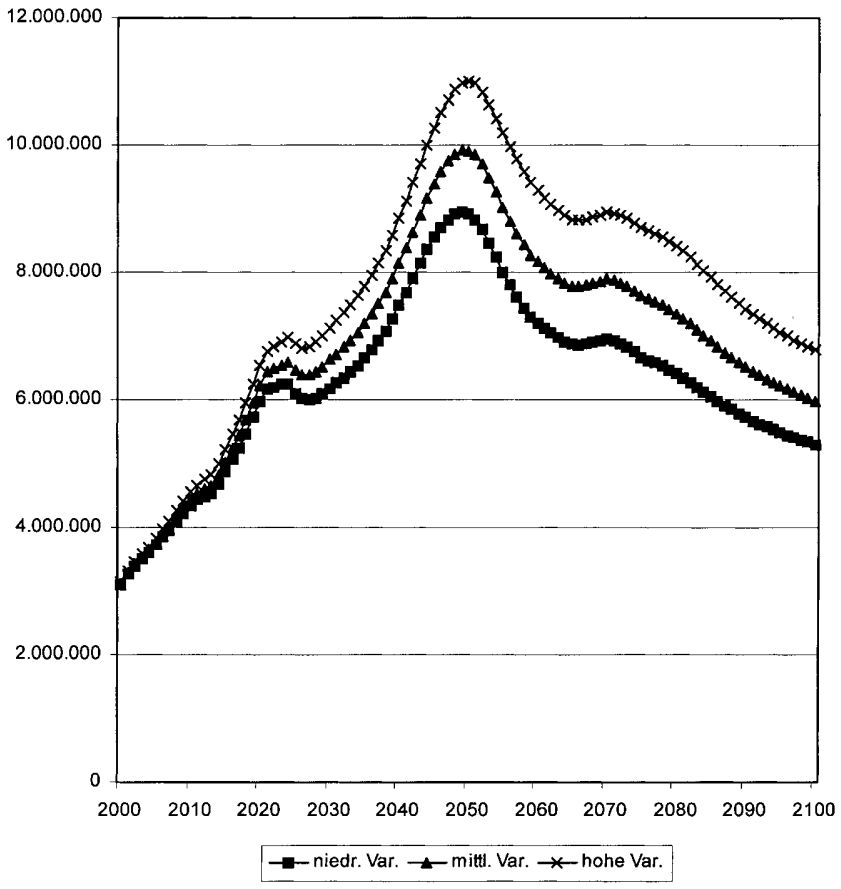

Abb. 10 Entwicklung der Zahl der 80-jährigen und älteren Bevölkerung in Deutschland in Abhängigkeit vom Anstieg der Lebenserwartung

der Säuglingssterblichkeit beruhte, stieg in der zweiten Hälfte vor allem die fernere Lebenserwartung im höheren Alter deutlich an. Dieser Anstieg war bei der weiblichen Bevölkerung stärker ausgeprägt als bei der männlichen. Während die durchschnittliche Lebenserwartung bei Männern von $1949 / 51$ bis 1997/99 um 15,3\% und bei Frauen um $17,7 \%$ anstieg, betrug die Zunahme der ferneren Lebenserwartung in diesem Zeitraum bei einem 80-jährigen Mann 31,9\% und bei einer 80 -jährigen Frau 50,3\%.

Wenn die fernere Lebenserwartung älterer Menschen auch zukünftig weiter ansteigt, dann wird sich diese Entwicklung auch in der Zahl der hochbetagten Menschen widerspiegeln. Im Jahr 2000 betrug die Zahl der 80-jährigen und älteren Bevölkerung rd. 3,1 Mio., die der 100-jährigen und älteren Bevölkerung schätzungsweise 9500. Bereits bis 2025 wird sich die Zahl der 80-jährigen und älteren Bevölkerung verdoppelt haben (Abb. 10). Die dann folgende, kurze Stagnation des Anstiegs beruht auf den Geburtenausfällen während bzw. vor allem zum Ende des Zweiten Weltkrieges. Die darauf folgenden geburtenstarken Nachkriegsjahrgänge werden bis zum Jahr $2050 \mathrm{zu}$ einem starken Anstieg der hochbetagten Bevölkerung führen, der in Abhängigkeit vom Verlauf der ferneren Lebenserwartung zu einer Zahl von 9 bis 11 Mio. Menschen im Alter von 80 Jahren und mehr führen wird. Das bedeutet, dass die hochbetagte Bevölkerung 


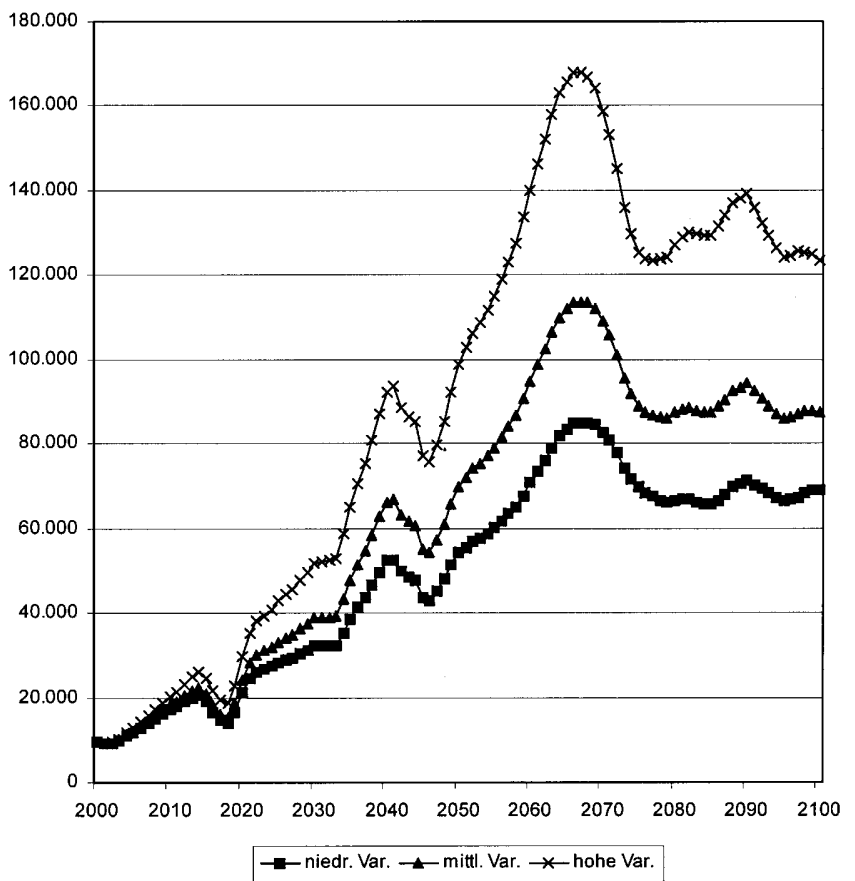

Abb. 11 Entwicklung der Zahl der 100-jährigen und älteren Bevölkerung in Deutschland in Abhängigkeit vom Anstieg der Lebenserwartung

Deutschlands bis zur Mitte des 21. Jahrhunderts um den Faktor 3 bis 3,5 zunehmen wird.

Bei der 100-jährigen und älteren Bevölkerung wird dieser Anstieg noch wesentlich stärker ausfallen (Abb. 11). Die Entwicklung der ferneren Lebenserwartung hat für die Besetzungszahlen dieser Altersgruppe eine noch wesentlich größere Bedeutung. Trotz des relativ geringen Zuwachses an Lebenserwartung in den hier berücksichtigten Varianten wird die Zahl der Hundertjährigen und Älteren auBerordentlich ansteigen, bis 2067/68 voraussichtlich auf 85000 in der unteren Variante und auf 167000 in der oberen Variante.

Bis zum Jahr 2050 wird der Anteil der 80-jährigen und älteren Bevölkerung von heute 3,8\% auf 13,4 bis $16,6 \%$ der Gesamtbevölkerung wachsen; der Anteil der 100-jährigen und älteren Bevölkerung wird in demselben Zeitraum von 0,01 auf 0,08 bis $0,15 \% \mathrm{zu}$ nehmen.

\section{Zusammenfassung}

Mit dem Begriff „demographische Alterung“ wird die Zunahme des Durchschnittsalters einer Bevölkerung bezeichnet. Sie lässt sich messen durch die Anteile der Altersgruppen am Bevölkerungsbestand, das Medianalter, den Altenquotienten sowie durch die Zahl und den Anteil der Betagten und Hochbetagten ( 80 Jahre und mehr). Die demographische Alterung hängt nicht nur von der Zunahme der Lebenserwartung vor allem im höheren Alter ab, sondern stärker noch vom niedrigen Niveau der Fertilität bzw. vom Rückgang der nachwachsenden jüngeren Altersgruppen, sowie vom Umfang der Einwanderungen Jüngerer aus dem Ausland.

Die demographische Alterung ist ein weltweites Phänomen, sie betrifft sowohl Industrie- als auch Entwicklungsländer. In den Industrieländern, insbesondere in Japan und Deutschland, ist die demographische Alterung besonders intensiv, in den USA ist sie infolge der höheren Fertilität weniger ausgeprägt als in Japan und Deutschland.

In Deutschland wird das Medianalter der Bevölkerung bis zum Jahr 2050 von 38 auf 52 und der Altenquotient ( $=$ Zahl der Personen im Alter von 60 und mehr auf 100 Menschen im Alter von 20-59) von 38,6 (1998) auf mehr als das Doppelte, wahrscheinlich auf 92 im Jahr 2050 zunehmen. Die Zunahme hängt von der Entwicklung der Fertilität, der Mortalität und der Migration ab. Zur Ermittlung der Intervallgrenzen für die künftige Entwicklung wurden in demographischen Simulationsrechnungen unterschiedliche Szenarien der Bevölkerungsentwicklung durchgerechnet. Alle Varianten zeigen, dass der Prozess der demographischen Alterung ein unabwendbares Phänomen ist, das gravierende Auswirkungen auf das Wirtschaftswachstum, den Arbeitsmarkt und die sozialen Sicherungssysteme haben wird.

Darüber hinaus werden zur Beschreibung des Alterungsprozesses in der Zukunft auch bestimmte Eck-Zahlen aus den Bevölkerungsvorausberechnungen herangezogen. Für Deutschland wurden z. B. von den Verfassern folgende Ergebnisse ermittelt:

1. In Deutschland nimmt die Zahl der 60-Jährigen und Älteren bis 2050 um rd. 10 Mio. zu.

2. Gleichzeitig geht die Zahl der 20- bis unter 60-Jährigen um rd. 16 Mio. zurück, und zwar auch dann, wenn man einen Überschuss der Einwanderungen jüngerer Menschen über die Auswanderungen von z. B. jährlich 170 Tsd. unterstellt, was dem bisherigen langfristigen Trend entspricht.

3. Am stärksten wächst die Gruppe der Hochbetagten. Die Zahl der 80-Jährigen und Älteren beträgt heute rd. 3 Mio., sie erhöht sich bis 2050 auf 8 bis 10 Mio.

4. Als Konsequenz aus der gegenläufigen Entwicklung - Wachstum der 60-Jährigen und Älteren und Schrumpfung der 20 bis unter 60-Jährigen nimmt der Altenquotient (= über 60-Jährige auf 100 Menschen im Alter von 20-60) von rd. 38 im Jahr 1998 auf 92 im Jahr 2050 zu (=Anstieg um den Faktor 2,4). 
Die Brisanz der demographischen Alterung beruht vor allem auf ihren Konsequenzen für die sozialen Sicherungssysteme. Wenn sich der Altenquotient mehr als verdoppelt, müssen - bei gleichbleibendem Versorgungsniveau - alle auf dem Umlageverfahren basierenden Zweige des Sozialen Sicherungssystems, d.h. vor allem die gesetzliche Renten- und Pflegeversicherung ihre Beitragssätze ebenfalls mindestens verdoppeln. Da dies nicht praktikabel ist, sind ein- schneidende Reformen des sozialen Sicherungssystems unumgänglich. Darüber hinaus werden als mögliche Lösungen die Erhöhung des Ruhestandsalters, die Anhebung der Frauenerwerbstätigkeit und die verstärkte Einwanderung jüngerer Menschen diskutiert, wobei diese Maßnahmen die demographische Alterung nicht beseitigen, sondern nur ihre Auswirkungen mildern.

\section{Literatur}

1. Birg H (1995) Population Projections for the 21st Century, Frankfurt/M u. New York

2. Birg H (1996) Die Weltbevölkerung Dynamik und Gefahren, München

3. Birg H (2000) 188 Millionen Einwanderer zum Ausgleich? In: Frankfurter Allgemeine Zeitung, Nr. 87, 12. April 2000, S 15

4. Birg H (2000) Perspektiven der Bevölkerungsentwicklung in Deutschland und Europa - Konsequenzen für die sozialen Sicherungssysteme, Vortrag bei der Sachverständigenanhörung des Bundesverfassungsgerichts in Karlsruhe am 4. Juli 2000, www.ibs.uni-Bielefeld.de.

5. Birg H (2001) Die demographische Zeitenwende - Der Bevölkerungsrückgang in Deutschland und Europa, München

6. Birg H, Flöthmann E-J (1996) Wanderungen älterer Menschen in den Regionen Nordrhein-Westfalens. Unveröffentlichter Forschungsbericht im Auftrag der Staatskanzlei des Landes Nordrhein-Westfalen, Bielefeld

7. Birg $\mathrm{H}$, Flöthmann E-J, Frein Th, Ströker K (1998) Simulationsrechnungen zur Bevölkerungsentwicklung in den alten und neuen Bundesländern im 21. Jahrhundert, IBS Materialien Bd. 45, Bielefeld

8. Birg H (2000) An approach for forecasting life expectancy and ist application in germany. In: Zeitschrift für Bevölkerungswissenschaft, Nr. 1, S 175198

9. Birg H, Flöthmann E-J (2001) Demographische Projektionsrechnungen für die Rentenreform 2000 - Methodischer Ansatz und Hauptergebnisse, IBS-Materialien Bd. 47A, und -Ergebnisse in Tabellenform, IBS Materialien Bd. 47B, Bielefeld

10. Bomsdorf E (1993) Generationensterbetafeln für die Geburtsjahrgänge 1923-1993. Bergisch-Gladbach, Köln
11. Bongarts J, Feeney G (2002) How long do we live? In: population and development review. No. 28, S 13-29

12. Bundesamt für Bauwesen und Raumordnung (2001) (Hrsg) Aktuelle Daten zur Entwicklung der Städte, Kreise und Gemeinden, Ausgabe 2000. Berichte des BBR, Bd. 8, Bonn

13. Bundesinstitut für Bevölkerungsforschung (1996) (Hrsg) Neue Sterbetafel des Verbandes der privaten Krankenversicherung. In: BiB-Mitteilungen, Nr. 1, S 18

14. Chesnais J-C (1992) The demographic transition-stages, patterns and economic implications. Oxford

15. Deutsche Bundesbank (1997) (Hrsg) Die Fiskalische Belastung zukünftiger Generationen - Eine Analyse mit Hilfe des Generational Accounting. In: Deutsche Bundesbank, Monatsbericht, S 17-30

16. Deutscher Bundestag (2002) (Hrsg) Schlussbericht der Enquete-Kommission „Demographischer Wandel Herausforderungen unserer älter werdenden Gesellschaft an den Einzelnen und die Politik“, Drucksache 14/8800, Berlin

17. Dinkel R, Höhn Ch, Scholz RD (1996) Sterblichkeitsentwicklung unter besonderer Berücksichtigung des Kohortenansatzes, München

18. Gärtner K (1990) Sterblichkeit nach dem Familienstand. In: Zeitschrift für Bevölkerungswissenschaft, 16 . Jg., Nr. 1, S 53-66

19. Gröner G (1996) Zur Entwicklung der Sterblichkeit in Baden-Württemberg 1985-1994. In: Baden-Württemberg in Wort und Zahl, Nr. 12, S 416-550

20. Hayflick L (1980) Biological aspects of aging. In: Preston S (Ed) Biological and social aspects of mortality and the length of life, Liège

21. Heigl A, Mai R (1998) Demographische Alterung in den Regionen der EU. In: Zeitschrift für Bevölkerungswissenschaft, Jg. 23, Nr. 3, S 293-317
22. Höhn Ch (1999) Die demographische Alterung - Bestimmungsgründe und wesentliche Entwicklungen. In: Höhn $\mathrm{Ch}$, Grünheid E. Demographische Alterung und Wirtschaftswachstum, Opladen, S 9-32

23. Höhn Ch, Pollard JH (1992) Persönliche Gewohnheiten und Verhaltensweisen und Sterblichkeitsunterschiede nach dem Familienstand in der Bundesrepublik Deutschland. In: Zeitschrift für Bevölkerungswissenschaft, 18. Jg., Nr. 4, S 415-433

24. Horiuchi S (1991) Adressing the affects of mortality reduction and population. In: Population Bulletin of the United Nations

25. Klein Th (1996) Mortalität in Deutschland - Aktuelle Entwicklungen und soziale Unterschiede. In: Zapf W, Schupp J, Habich R (Hrsg) Lebenslagen im Wandel: Sozialberichterstattung im Längsschnitt, Frankfurt/New York, S 366-377

26. Limbacher M (1986) Regionale Mortalitätsanalyse 1979-1983. In: Statistische Rundschau für das Land Nordrhein-Westfalen, Nr. 2, S 75-88

27. Linke W (1990) Differentielle Sterblichkeit nach Berufen. In: Zeitschrift für Bevölkerungswissenschaft, 16. Jg., Nr. 1, S 29-51

28. Mayer KU, Staudinger U (1992) Berliner Altersstudie: im Mittelpunkt die alten Alten. In: Spektrum der Wissenschaft, S 101-116

29. Neubauer G (1986) Kleinräumige Unterschiede der Sterblichkeit in Bayern und deren mögliche Ursachen. Hochschule der Bundeswehr, Institut für Volkswirtschaftslehre, Diskussionsbeiträge, Nr. 28, München

30. Oeppen J, Vaupel JW (2002) Broken limits to life expectancy. In: Science, No. 296, p 1029-1031

31. Olshansky SJ (1988) On forecasting mortality. In: The Milbank Quarterly, Vol. 66, No. 3, S 482-530

32. Rose MR (1989) Evolutionary biology of aging. Oxford University Press 
33. Ruzicka L, Wunsch G, Kane P (Ed) (1989) Differential Mortality, Oxford

34. Schmithals B, Schütz EU (1995) Herleitung der DAV-Sterbetafel 1994 R für Rentenversicherungen. In: Deutsche Gesellschaft für Versicherungsmathematik (Hrsg) Blätter, Bd. XXII, Heft 1, S 31-69

35. Statistisches Bundesamt: Fachserie 1, Reihe 1, Gebiet und Bevölkerung, Wiesbaden, jährlich

36. Statistisches Bundesamt (1969) (Hrsg) Spezielle Sterbetafeln 1960/62. Fachserie A. Reihe 2, Sonderbeitrag, Stuttgart/Mainz

37. Statistisches Bundesamt (2000) (Hrsg) 9. Koordinierte Bevölkerungsvorausberechnung, Wiesbaden
38. Thatcher AR, Kannisto V, Vaupel JW (1988) The force of mortality at ages 80 to 120. Odense Monographs on Population Aging 5, Odense University Press

39. United Nations (Ed) (1998) Bericht über die menschliche Entwicklung, Bonn

40. United Nations, Population Division (2000) Replacement migration: Is it a solution to declining and ageing populations? New York

41. United Nations, Population Division (2001) World population prospects the 2000 revision. New York
42. Valkonen R (1998) Die Vergrößerung der sozioökonomischen Unterschiede in der Erwachsenenmortalität durch Status und deren Ursachen. In: Zeitschrift für Bevölkerungswissenschaft, 23. Jg., Nr. 3, S 263-292

43. Vaupel JW, Lundström H (1996) The future of mortality at older ages in developed countries. In: Lutz W (Hrsg) The future population of the world - what can we assume today? Revised Edition, Laxenburg, S 278296

44. White KM (2002) Longevity advances in high-income countries, 1955-1996. In: Population and Development Review, No. 28, S 59-76 